\title{
Die Beziehung zum Kunden auf eine neue Ebene bringen
}

\author{
Das Value Proposition Canvas erfreut sich als Management-Tool großer \\ Beliebtheit, unter anderem zur Entwicklung neuer Geschäftsmodelle. Im Key \\ Account Management birgt das Instrument großes Potenzial zur Konfiguration \\ individueller Lösungen für Schlüsselkunden.
}

Im Key Account Management (KAM) werden die strategisch wichtigen Kunden eines Unternehmens systematisch bearbeitet. Dazu gehören passgenaue, kundenindividuelle Lösungen und Argumentationen. Um diese zu entwickeln, sind Key Account Manager zusammen mit ihren Teams herausgefordert. Den Schlüsselkunden und seine Bedürfnisse müssen sie dabei stets im Blick behalten und ihn sowie die wichtigen Entscheider gleich bei den Überlegungen einbeziehen. Hier ist das Value Proposition Canvas ein hilfreiches Instrument. Canvas-Ansätze erfreuen sich im Management großer Beliebtheit. In der Regel werden sie zum Aufbau neuer Geschäftsmodelle und für deren Ausgestaltung genutzt. Im Vertrieb werden sie heute noch eher selten eingesetzt. Canvas ist das englische Wort für Leinwand. Es kann auch als Arbeitsgrundlage genutzt werden, eine Plakatwand, auf der man in einem kreativen Teamprozess arbeitet, schreibt, zeichnet oder klebt. Als Workshopmethode im Management wurden Canvas-Ansätze von Alexander Osterwalder und anderen Forschern zuerst als „Business Modell Canvas“ entwickelt und später durch das „Value Proposition Canvas“ ergänzt.

\section{Kompakt}

- Das Value Proposition Canvas ermöglicht das Entwickeln individueller Lösungen für Key Accounts eines Unternehmens im KAM-Team oder sogar gemeinsam mit dem Schlüsselkunden.

- Das plakative Vorgehen setzt kreative Energien frei und dient dem Key Account Manager zugleich als Koordinations- und Führungsinstrument.

- Der Aufbau des Canvas stellt sicher, dass Lösungen genau zum Kunden und seinen Bedürfnissen passen und hilft zugleich, die Argumente zu schärfen und überzeugender zu vermitteln.

\section{Value Proposition Canvas im KAM}

Das Value Proposition Canvas, dessen Begriffe in der Abbildung bereits auf das KAM angepasst sind, besteht aus zwei Teilen: dem so genannten Kundenprofil, hier Key-AccountProfil genannt, und der Value Map.

\section{„Ein wichtiger Erfolgsfaktor der Analyse des Key Accounts ist die Kenntnis des Buying Centers, also der entscheidungsrelevanten Personen beim Schlüsselkunden."}

Sinnvollerweise startet man die Betrachtung und Arbeit mit dem Canvas von rechts nach links. Der rechte Bereich ist der des Key-Account-Profils, der in drei Themen unterschieden wird. Erstens: Welche Aufgaben hat ein Key Account, das heißt, was muss der Key Account tun, um in seinem Markt erfolgreich zu sein? Zweitens: Welche Ziele oder Ergebnisse strebt er dabei an? Und schließlich geht es drittens um die Frage, wo der Key Account bei seinen Aufgaben Probleme hat. Im Kundenprofil liegt der Fokus darauf, den Kunden in seinem Geschäftsmodell umfassend zu verstehen, inklusive seiner Ambitionen und Herausforderungen.

Die Value Map auf der linken Seite der Abbildung ist das Pendant zum Kundenprofil. Damit ermittelt der Anbieter, wie eine kundenindividuelle Lösung aussehen sollte und er argumentieren will. Dazu wird das passende Leistungsangebot, also Produkte und Dienstleistungen, entwickelt. Neben der Lösung geht es um die konkreten Beiträge des Anbieters, die dem Kunden helfen, seine Ziele zu erreichen. Sie heißen im Original „Gewinnerzeuger“. Und man erarbeitet, wo man 
dem Schlüsselkunden helfen kann, Probleme zu lösen, im Original „Problemlöser“ genannt.

\section{Das Vale Proposition Canvas im Key Account Management}

Key Account Management ist die systematische Analyse und das Management der strategisch wichtigsten Kunden eines Unternehmens, um damit Wettbewerbsvorteile zur erzielen. Das Key-Account-Profil ist ein wertvolles Tool für die Analyse des Kunden und seiner Aufgaben, die Value Map ist ein Hilfsmittel, um passgenaue Lösungen mit den richtigen Argumenten zu entwickeln.

Ein wichtiger Erfolgsfaktor der Analyse des Key Accounts ist die Kenntnis des Buying Centers, also der entscheidungsrelevanten Personen beim Schlüsselkunden. In der BuyingCenter-Analyse analysiert ein professionelles KAM, wer für die Geschäftsbeziehung relevant ist, welche Bedürfnisse und Ziele diese Personen haben, welche Rolle sie spielen, welche Macht sie in der Geschäftsbeziehung haben und so weiter. Diese Informationen sind Ausgangsbasis des Kundenprofils für einen bestimmten Key Account. Folgende Fragen können helfen, diesen Bereich in einem Workshop mit Leben zu füllen:

- Welche Personen sind Teil des Buying Centers des Key Accounts?

- Welche Aufgaben haben diese Personen im Rahmen ihrer Jobs im Key-Account-Unternehmen?

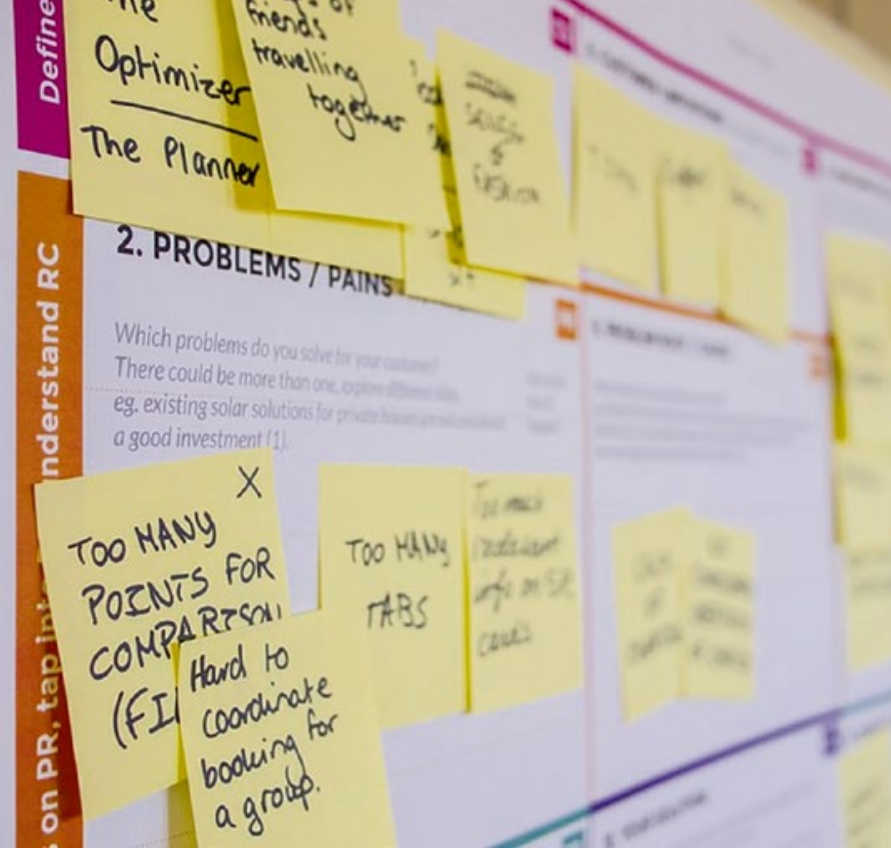

- Welche Aufgaben haben diese Personen, die sich aus den Rollen im Buying Center (zum Beispiel Entscheider, Beeinflusser, Nutzer, Einkäufer) ergeben?

- Welche sozialen und emotionalen Interessen (zum Beispiel Macht, Anerkennung, Status) haben diese Personen?

- Was tun sie, um diese Interessen zu verfolgen?

Mit einem guten Verständnis der Aufgaben des Key Accounts beziehungsweise der Personen im Buying Center lassen sich die Ziele herausarbeiten:

- Welche konkreten Ziele ergeben sich für die Personen im Buying Center aus ihrer Verantwortung?

Abb. Aufbau des Value Proposition Canvas

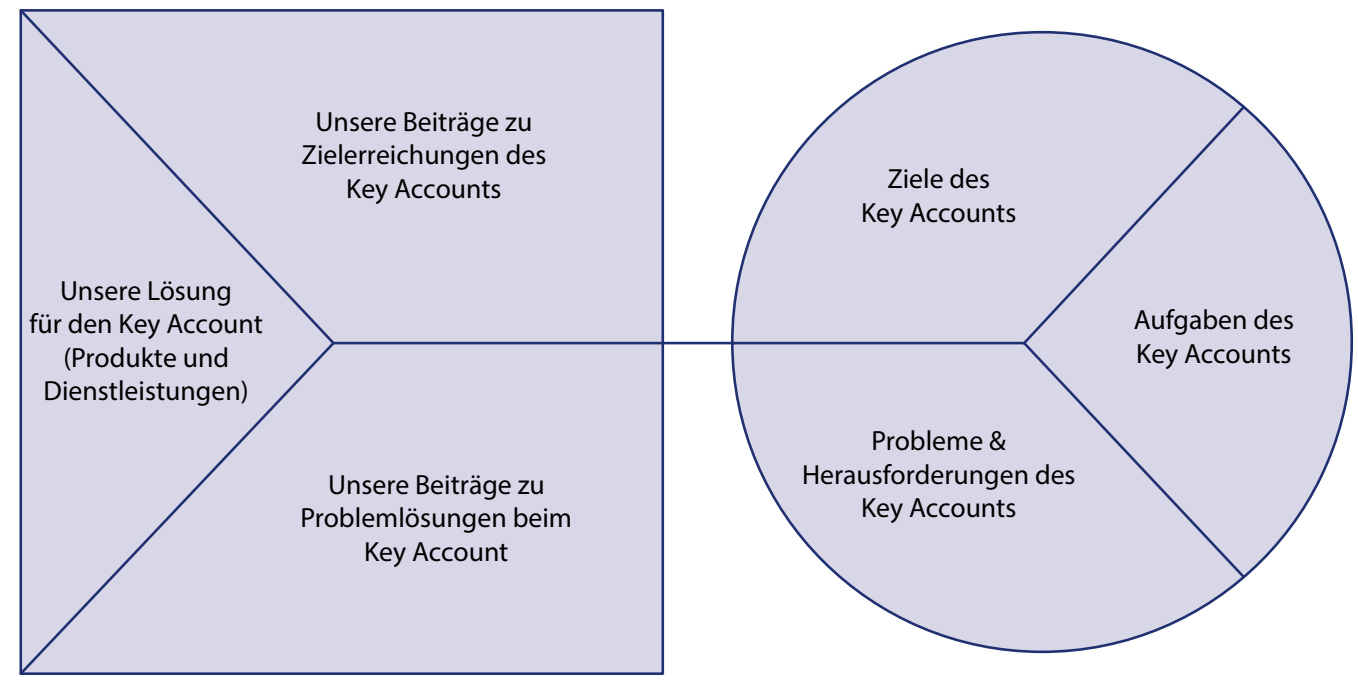

Key Account Value Map

Key-Account-Profil 


\section{Empfehlungen}

- Die Qualität der Ergebnisse steigt, wenn alle Beteiligten ein solides Verständnis über den Key Account und sein Geschäft haben. Hier ist es hilfreich, wenn die Inhalte eines KAM-Plans, die heute zum Standard gehören sollten (zum Beispiel Analyse des Key Accounts, insbesondere seiner Makro-/Mikroumwelt, den Buying Centers, SWOT, das aktuelle Geschäft bei diesem Kunden) vorher an alle Workshopteilnehmer ausgegeben werden und die Teilnehmer sich gut vorbereiten.

- Alle relevanten Personen des Anbieterunternehmens, die einen Beitrag zum Erfolg dieses Kunden leisten, idealerweise das KAM-Team, sollten teilnehmen.

- Der Key Account Manager sollte sich selbst gut vorbereiten und erste Inhalte des Canvas im Kopf haben, um den Workshop souverän moderieren zu können. Prinzipiell kann man die Moderation auch delegieren. Allerdings erweist sich ein gut geführter Workshop auch als wertvolles Tool für das Team-Management im KAM. Das ist eine Chance für einen Key Account Manager, seinen Führungsanspruch und seine Kompetenz für diesen Kunden deutlich zu machen. Zugleich ist die Methode eine wertvolle Basis, um alle Teammitglieder am Prozess zu beteiligen und auch ihre zukünftigen Beiträge für diesen Key Account verbindlich festzulegen.

- In besonderen Fällen, in der Regel bei einer sehr vertrauensvollen Geschäftsbeziehung, kann man auch Vertreter des Key Accounts selbst mit einbeziehen und so die Geschäftsbeziehung gemeinsam kooperativ weiterentwickeln. Dennoch ist es ratsam, sich als Anbieter klar in die Verantwortung zu begeben, weil nach allen Erfahrungen der Anbieter die Geschäftsbeziehung kundenorientiert führen sollte und nicht umgekehrt.

- Die Arbeit im Workshop erfolgt an der Plakatwand, dem Canvas, unter Nutzung von Post-its, gegebenenfalls auch Skizzen und so weiter. In diesem Prozess darf es ruhig sehr interaktiv und zuweilen auch etwas chaotisch zugehen. Der Kreativität sollte hier Raum und Zeit gegeben werden. Später kann man das Ergebnis gemeinsam reduzieren und fokussieren sowie Details ausarbeiten. Das fertige Ergebnis sollte in der KAM-Plan eingepflegt werden, konkrete To-dos müssen zwingend abgeleitet und mit Verantwortlichen und entsprechenden Deadlines versehen werden.
- Welche Ziele ergeben sich aus ihren Rollen im Buying Center?

- Welche Ziele ergeben sich aus ihren persönlichen, sozialen und emotionalen Interessen?

- Sodann geht es nach dem gleichen Muster um die Probleme:

- Welche Probleme und Herausforderungen haben Personen im Buying Center in ihren Jobs?

- Welche Probleme und Herausforderungen ergeben sich aus ihren Rollen im Buying Center?

- Welche Probleme entstehen aus ihren persönlichen, sozialen und emotionalen Interessen?

Alle Ergebnisse und Ideen werden in Form von Post-its in die entsprechenden Bereiche der Leinwand geklebt und können gegebenenfalls durch Symbole oder Skizzen ergänzt werden. Die Post-its haben dabei den Vorteil, dass sie leicht neu geschrieben und ersetzt oder neu positioniert werden können, was durchaus häufig vorkommt.

\section{„Idealerweise steht jeder relevanten Kundenaufgabe eine Leistung gegenüber, die dem Key Account hilft, Ziele zu erreichen und/oder Probleme zu lösen."}

Sollten die Ausarbeitungen an dieser Stelle zu umfangreich werden, kann man alle Erkenntnisse für die weitere Arbeit priorisieren. Dabei ist es trotzdem hilfreich, wenn man zunächst eine Vollständigkeit des Kundenprofils für den Key Account anstrebt und erst dann selektiert oder priorisiert.

Von der Seite des Key-Account-Profils geht es auf die Value Map, die mit einer kreativen Zusammenstellung der Produkte und Dienstleistungen startet. Hier geht es darum, Leistungen zu listen, die dem Key Account beziehungsweise den entscheidenden Personen aus dem Buying Center helfen, die Aufgaben zu bewältigen, ihre angestrebten Ziele zu erreichen und die auf dem Weg liegenden Probleme zu lösen. Nach dem kreativen Sammeln der Leistungen steht die Formulierung und Schärfung der Argumentation im Vordergrund: Welche Produkte und Dienstleistungen helfen dem Key Account konkret, seine Ziele zu erreichen? Welche helfen, die Probleme zu lösen?

Das fertige Canvas sollte auf Konsistenz überprüft und gegebenenfalls ergänzt und erweitert werden. Idealerweise steht jeder relevanten Kundenaufgabe eine Leistung gegenüber, die dem Key Account hilft, Ziele zu erreichen und/oder Probleme zu lösen. Sind die Ergebnisse zu wenig neu und innovativ oder dem Mitbewerb zu ähnlich, sollte man weiter- 
arbeiten. Dazu können weitere Erkenntnisse recherchiert und zusätzliche Experten hinzugezogen werden.

Vollständig ausgearbeitet ermöglicht das Canvas eine kundenorientierte Argumentation. Für einen Anlagenbauer könnte das so aussehen:

- Durch unsere Anlage (Produkt/Zielbeitrag) sind Sie in der Lage, die gewünschte Kapazität von x Tonnen pro Tag (Ziele) zu produzieren (Aufgabe).

- Durch unser Servicepaket (Dienstleistung/Problemlösung) garantieren wir Ihnen die Sicherheit (Problem) eines ausfallsicheren Ablaufes (Aufgabe).

- Das optionale Finanzierungsangebot der Anlage (Dienstleistung/Zielbeitrag) bietet Ihnen die finanzielle Flexibilität (Ziele) zur Bewältigung anderer Projekte (Aufgabe).

- Mit unserem Vorschlag eines Betreibermodels (Produkt/ Problemlösung) schaffen Sie freie Kapazitäten bei Ihren Mitarbeitenden (Problem) für das angestrebte Wachstum (Ziele) im Rahmen Ihrer Markterweiterung (Aufgabe).

Die Nutzung des Canvas-Ansatzes erfolgt idealerweise in einem Workshop. Für den Key Account Manager ist das Instrument auch persönlich ein wertvolles Hilfsmittel zur Analyse des Key Accounts und zur Ausarbeitung einer kundenindividuellen Lösung mit einen KAM-Team. Aus der Erfahrung lohnt es sich, einige Hinweise für den Einsatz in der Praxis zu beachten (siehe Empfehlungen).

\section{„Für den Key Account Manager ist das Instrument ein wertvolles Hilfsmittel zur Ausarbeitung einer kundenindividuellen Lösung mit einen KAM-Team."}

\section{Erarbeitung einer kundenindividuellen Lösung}

Das Value Proposition Canvas bereichert das Instrumentarium im KAM. Es hilft insbesondere den Key Account Managern, zusammen mit ihrem Team den Key Account richtig zu verstehen und daraus sehr fokussiert eine kundenindividuelle Lösung zu erarbeiten. Der Teamprozess im Workshop, gegebenenfalls sogar zusammen mit Vertretern des Kunden, kann dabei auch neue, innovative Ideen erzeugen, die Kunden überraschen, die Geschäftsbeziehung auf eine neue Ebene befördern und so die Wettbewerbsvorteile des Anbieters stärken oder ausbauen. Damit am Ende des kreativen Prozesses die Ergebnisse auch umgesetzt werden, bedarf es verbindlicher Aufgaben und der Führung durch den Key Account Manager.

\section{Literatur}

Belz, C., Müllner, M., Zupancic, D. (2015): Spitzenleistungen im Key Account Management, München

Osterwalder et al. (2011): Business Model Generation: Ein Handbuch für Visionäre, Spielveränderer und Herausforderer, Frankfurt/ New York

Osterwalder et al. (2015): Value Proposition Design: Entwickeln Sie Produkte und Services, die ihre Kunden wirklich wollen, Frankfurt/ New York

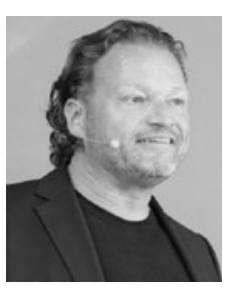

Autor

Prof. Dr. Dirk Zupancic ist Inhaber der DZP $\mathrm{GmbH}$, Aufsichtsrat und habilitierter Privatdozent an der Universität St. Gallen. Er berät, trainiert und coacht Führungskräfte, Vertriebsfachleute und Unternehmen.

E-Mail: dirk.zupancic@dzp-consulting.com

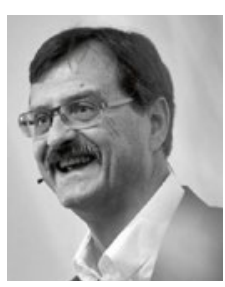

Prof. Dr. Christian Belz ist emeritierter Professor der Universität St. Gallen. Seine Schwerpunkte in der Forschung und in Unternehmensprojekten umfassen Industriemarketing, Leistungs- und Kundenmanagement.

E-Mail: christian.belz@unisg.ch

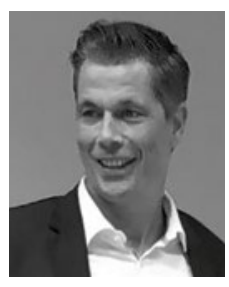

Dr. Markus Müllner ist Key-Account-Management-Berater und Führungscoach bei internationalen Unternehmen sowie Dozent an renommierten Universitäten und Business Schools.

E-Mail: markus.muellner@new-spirit.ch

\section{SpringerProfessional.de}

Fischer, H., Rittmeier, F., Strothmann, T., Schwenniger, N.: Partizipation von Beschäftigten in der Gestaltung einer digitalisierten Arbeitswelt 4.0 mittels einer Canvas-Methode, in: Bosse, C. K., Zink, K. J.: Arbeit 4.0 im Mittelstand, Berlin/Heidelberg 2019

www.springerprofessional.de/link/17492356 